\title{
On migration load of seeds and pollen grains in a local population
}

\author{
Xin-Sheng $\mathrm{Hu}^{1}$ and Bailian $\mathrm{Li}^{2}$ \\ ${ }^{1}$ Department of Forest Sciences, University of British Columbia, 3rd floor, 3041-2424 Main Mall, Vancouver, Canada BC V6T 1Z4; \\ ${ }^{2}$ Department of Forestry, North Carolina State University, PO Box 8002, Raleigh, NC 27695, USA
}

\begin{abstract}
We have extended Wright's model of migration load to hermaphrodite plants showing variation at a single locus with two alleles. The model incorporates independent migration of seeds and pollen grains, the selection at both the haploid gametophyte and the diploid sporophyte stages, and a mixed mating system. The analytical relations between migration load and migration rate of seeds and pollen grains are explicitly formulated. The results show that under certain conditions, seed flow can have a more effect on migration load than pollen flow. Pollen selection at the gametophyte
\end{abstract}

stage cannot substantially affect the migration load at the sporophyte stage. Selection at the diploid sporophyte stage is critical in determining the migration load of pollen grains. The relative migration loads of pollen versus seeds can be approximately estimated in predominantly outcrossing populations by the ratio of pollen flow to twice the seed flow, when the selection coefficient $\left(s_{\mathrm{T}}\right)$ is greater than, or approximately equal to, the migration rate $(\tilde{m})$.

Heredity (2003) 90, 162-168. doi:10.1038/sj.hdy.6800212

Keywords: migration load; seed flow; pollen flow; pollen selection; ovule selection

\section{Introduction}

Gene flow can homogenize the genetic divergence among populations. On the one hand, effects of genetic drift in small local populations can be effectively reduced when the average number of migrants is greater than one (Wright, 1969), beneficial immigrant genes can shift local populations to a higher fitness peak (Barton and Whitlock, 1997). On the other hand, gene flow between populations adapted to different environments can cause maladaptation in a recipient population, resulting in migration load, a reduction in population fitness. If the migration rate is much greater than the selection coefficient, migrant alleles can even swamp out locally adaptive alleles (Wright, 1969).

In hermaphrodite plants, interpopulation gene flow is mediated through either seed or pollen, or both. Pollen flow includes successful fertilization of ovules and establishment of the resulting seeds. Seed flow includes germination, survival, and growth to the next adult generation. In reality, more pollen grains are often deposited on the stigma than are necessary to fertilize all ovules in a flower, creating an opportunity for selection among pollen grains before zygote formation (eg Snow and Spira, 1991, 1996; Björkman, 1995; Quesada et al, 1996; Niesenbaum, 1999). In plants, genes can be expressed at both the gametophyte and the sporophyte stages (Haldane, 1932; Charlesworth and Charlesworth, $1992 a, b)$. Approximately $60 \%$ of the structural genes that are expressed in the sporophytic portion of angiosperm

Correspondence: X-S Hu, Department of Forest Sciences, University of British Columbia, 3rd Floor, 3041-2424 Main Mall, Vancouver, Canada BCV6T 1Z4. E-mail: xhu@interchange.ubc.ca

Received 12 April 2002; accepted 26 September 2002 life cycle are also expressed and potentially subject to selection in the pollen (Mulcahy et al, 1996). The significance of pollen competition in natural populations has been extensively studied in many plant species, although its importance remains controversial (eg Mitchell, 1997; Kalla and Ashman, 2002).

Also, a single ovule in plants can contain more than one embryo (polyembryony), leading to selection among embryos sired by different pollen grains (eg Sorensen, 1982; Charlesworth and Charlesworth, 1987; Charlesworth, 1989a; Kärkkäinen and Savolainen, 1993). There are typically more ovules per flower and/or flowers per plant than matured seed produced. This implies the existence of selection when the seed parents abort the excess ovules and/or flowers (Charlesworth, 1989b). Although ovule-removal experiments show that ovules that would normally abort can develop into seeds after removal of other ovules, these seeds often have a lower vigour compared with control seeds (eg Casper, 1988; Stephenson and Winsor, 1986; Rocha and Stephenson, 1991; Melser and Klinkhamer, 2001). Like pollen selection, ovule selection cannot be excluded in natural populations. Therefore, a model of migration load should take into account the impact of selection at the gametophyte stage in addition to that at the sporophyte stage.

Since the distinct biological processes involved in gene flow via seed and pollen, the migration load generated by these processes could be different. Like the genes in resident pollen and ovules, the genes in migrant pollen grains can be affected by natural selection at both the gametophyte and the sporophyte stages because there is a connection between these two stages of life-phases. Thus, selection on the genes from immigrant pollen grains could also affect the variability in fitness at the 
sporophyte stage. In contrast to the case of pollen flow, the migration load of seeds occurs only at the sporophyte stage since migrant seeds have been formed in donor populations. Thus, elucidation of the relative migration loads of seeds versus pollen grains can reveal insights into the consequence of these two distinct biological processes.

Wright (1977, p 485-486) first studied the theory of migration load in a very general case and showed that its magnitude depends on relative migration rates and selection coefficients. In Wright's model of migration load, selection refers to that occurring at the sporophyte stage, and migration refers to diploid dispersal (seed flow here). The theory is not very appropriate for hermaphrodite plants, where haploid pollen flow and selection at the gametophyte stage can occur. Evidences for migration load have been recorded (see review by Lenormand, 2002), and the effects of migration on evolution of recipient populations have also been implied in many studies (eg Barton and Gale, 1993, p 30; Burt, 1995; Ellstrand et al, 1999; Barton, 2001; Whitlock, 2002). However, the theory of migration load has not been extensively developed since Wright's pioneer work. In a relevant study, Charlesworth and Charlesworth (1992a) examined the effects of selection at the gametophyte (pollen) stage on mutational load. However, compared with the mutation load, the migration load could be more substantial since plants are subject to much greater inputs of migrants compared with recent mutants (Waser and Price, 1989; Mulcahy et al, 1996). In a recent study, Lankinen and Skogsmyr (2001) proposed a phenotypic model of pollen competition. In neither of these studies are seed flow and ovule selection considered.

The purpose of this article is to develop further population genetic theory required for understanding the relative migration loads attributable to seed and pollen flow. In the proposed model, pollen and seed migration and selection at both the gametophyte and the sporophyte stages are taken into account. We give analytical relations between migration load and seeds and pollen migration rates, and explore the inferences that can be drawn about the relative migration loads of seeds and pollen grains.

\section{The model}

\section{General assumptions}

The model deals with a local population of hermaphrodite plants, with immigration of pollen and seeds from a 'migrant pool'. A single locus is considered with two alleles, denoted by $A$ and $a$. Mutation rate is assumed to be much smaller than migration rate or selection coefficient, and hence not included. The modeling process is based on the sequence of events in a plant life cycle: adult generation $t$, gamete (pollen and ovules) formation, pollen flow, gametophyte selection, mating, seed formation, seed flow, sporophyte selection, and adult generation $t+1$.

Deterministic and random processes are considered separately. Genetic drift is ignored when the local population size is sufficiently large, but considered as a random process when the local population size is small. In each case, the gene frequencies in migrant pollen grains or seeds are held constant in each generation, denoted by $Q$ for allele $A$ and $1-Q$ for allele $a$. The genotype frequencies in migrant seeds are assumed as $Q^{2}+F Q(1-Q)$ for $A A, 2(1-F) Q(1-Q)$ for $A a$, and $(1-Q)^{2}+F Q(1-Q)$ for $a a$, where $F$ is the inbreeding coefficient in the migrant seed pool. When $F$ is equal to zero, the genotype frequencies in migrant seeds follow Hardy-Weinberg equilibrium. The allele frequencies in ovules before combination with pollen grains or in pollen grains before dispersal are assumed to be the same as those at the preceding adult generation (Wright-Fisher's model).

\section{Deterministic process}

Let $p(t)$ and $q(t)$ be the frequencies of alleles $A$ and $a$ in adults at generation $t$, respectively. After pollen flow, the frequency of allele $A$ in pollen grains, denoted by $p_{\mathrm{P}}(t+1)$, is

$$
p_{\mathrm{P}}(t+1)=m_{\mathrm{P}} Q+\left(1-m_{\mathrm{P}}\right) p(t)
$$

where $m_{\mathrm{P}}$ is the migration rate of pollen. According to the above general assumptions, the frequency of allele $A$ in ovules, denoted by $p_{\mathrm{O}}(t+1)$ at generation $t+1$, remains the same as that of the preceding generation, that is,

$$
p_{\mathrm{O}}(t+1)=p(t)
$$

Let the selection coefficients at the gametophyte stage be 1 and $1-s_{\mathrm{P}}$ for alleles $A$ and $a$ in pollen grains, and 1 and $1-s_{\mathrm{O}}$ for alleles $A$ and $a$ in ovules, respectively. After selection at the gametophyte stage, the frequencies of allele $A$ in pollen and ovules, denoted respectively by $p_{\mathrm{P}}^{*}(t+1)$ and $p_{\mathrm{O}}^{*}(t+1)$, become

$$
\begin{aligned}
p_{\mathrm{P}}^{*}(t+1)= & p_{\mathrm{P}}(t+1)+s_{\mathrm{P}} p_{\mathrm{P}}(t+1) q_{\mathrm{P}}(t+1) \\
& +O\left(s_{\mathrm{P}}^{2}\right) \\
p_{\mathrm{O}}^{*}(t+1)= & p_{\mathrm{O}}(t+1)+s_{\mathrm{O}} p_{\mathrm{O}}(t+1) q_{\mathrm{O}}(t+1) \\
& +O\left(s_{\mathrm{O}}^{2}\right)
\end{aligned}
$$

where $q_{\mathrm{P}}(t+1)$ and $q_{\mathrm{O}}(t+1)$ are the frequencies of allele $a$ in pollen grains and ovules before selection, respectively.

Consider the ordered genotypes, such as $A A$, where the first allele is assumed to come from pollen and the second from an ovule. For simplicity, denote the frequencies of ordered genotypes $A A, A a, a A$, and $a a$ in adults, respectively, by $P_{11}(t), P_{12}(t), P_{21}(t)$, and $P_{22}(t)$ at generation $t$. Let $\alpha$ be the proportion of selfing and $1-\alpha$ be its counterpart. For the selfing component, the genotype frequencies, denoted by subscript I (Wright, 1969, p 244), can be written as

$$
\begin{aligned}
\mathbf{P}_{\mathrm{I}}(t+1) & \\
\quad= & \left(\begin{array}{cccc}
1 & 1 / w & 1 / w & 0 \\
0 & \left(1-s_{\mathrm{O}}\right) / w & \left(1-s_{\mathrm{O}}\right) / w & 0 \\
0 & \left(1-s_{\mathrm{P}}\right) / w & \left(1-s_{\mathrm{P}}\right) / w & 0 \\
0 & \left(1-s_{\mathrm{O}}-s_{\mathrm{P}}\right) / w & \left(1-s_{\mathrm{O}}-s_{\mathrm{P}}\right) / w & 1
\end{array}\right) \mathbf{P}(t)
\end{aligned}
$$

where $\quad w=2\left(2-s_{\mathrm{O}}-s_{\mathrm{P}}\right), \quad \mathbf{P}_{\mathrm{I}}(t+1)=\left[P_{11 . \mathrm{I}}(t+1)\right.$, $\left.P_{12 . \mathrm{I}}(t+1), P_{21 . \mathrm{I}}(t+1), P_{22 . \mathrm{I}}(t+1)\right]^{\prime}, \quad$ and $\mathbf{P}(t)=\left[P_{11}(t)\right.$, $\left.P_{12}(t), P_{21}(t), P_{22}(t)\right]^{\prime}$.

For the random mating component, the genotype frequencies, with subscript $\mathrm{R}$, are $P_{11 . \mathrm{R}}(t+1)=$ $p_{\mathrm{P}}^{*}(t+1) p_{\mathrm{O}}^{*}(t+1), P_{12 . \mathrm{R}}(t+1)=p_{\mathrm{P}}^{*}(t+1) q_{\mathrm{O}}^{*}(t+1), P_{21 . \mathrm{R}}$ $(t+1)=q_{\mathrm{P}}^{*}(t+1) p_{\mathrm{O}}^{*}(t+1)$, and $P_{22 . \mathrm{R}}(t+1)=q_{\mathrm{P}}^{*}(t+1)$ 
$q_{\mathrm{O}}^{*}(t+1)$. Similarly, we can obtain

$$
\begin{aligned}
\mathbf{P}_{\mathrm{R}}(t+1) & =\left(\begin{array}{cccc}
p^{2}(t) & -p(t) & p^{2}(t) q(t) & p^{2}(t) q(t) \\
0 & -q(t) & -p^{2}(t) q(t) & p(t) q^{2}(t) \\
0 & p(t) & p(t) q^{2}(t) & -p^{2}(t) q(t) \\
q^{2}(t) & q(t) & -p(t) q^{2}(t) & -p(t) q^{2}(t)
\end{array}\right) \\
& \times\left(\begin{array}{c}
1 \\
m_{\mathrm{P}}(p(t)-Q) \\
s_{\mathrm{O}} \\
s_{\mathrm{P}}
\end{array}\right)
\end{aligned}
$$

where $\mathbf{P}_{\mathrm{R}}(t+1)=\left[P_{11 . \mathrm{R}}(t+1), \quad P_{12 . \mathrm{R}}(t+1), P_{21 . \mathrm{R}}(t+1)\right.$, $\left.P_{22 . R}(t+1)\right]^{\prime}$. Therefore, the genotype frequency in seeds after mixed mating is

$$
\begin{gathered}
P_{i j . \mathrm{S}}^{*}(t+1)=\alpha P_{i j \mathrm{I}}(t+1)+(1-\alpha) P_{i j . \mathrm{R}}(t+1) \\
(i, j=1,2)
\end{gathered}
$$

Let $m_{\mathrm{S}}$ be the migration rate of seeds per generation. After seed flow, the genotype frequencies in seeds, denoted by $P_{i j . S}^{* *}(t+1)(i, j=1,2)$, are given by

$$
\begin{aligned}
\left(\begin{array}{c}
P_{11 . S}^{* *}(t+1) \\
P_{12 . S}^{* *}(t+1) \\
P_{21 . S}^{* *}(t+1) \\
P_{22 . S}^{* * .5}(t+1)
\end{array}\right)= & \left(\begin{array}{cc}
Q^{2}+F Q(1-Q) & P_{11 . S}^{*}(t+1) \\
(1-F) Q(1-Q) & P_{12 . S}^{*}(t+1) \\
(1-F) Q(1-Q) & P_{21 . S}^{*}(t+1) \\
(1-Q)^{2}+F Q(1-Q) & P_{22 . S}^{*}(t+1)
\end{array}\right) \\
& \times\left(\begin{array}{c}
m_{\mathrm{S}} \\
1-m_{\mathrm{S}}
\end{array}\right)
\end{aligned}
$$

Suppose that the fitness of a diploid genotype is equal to the product of the fitness for each allele (multiplicative viability model). Let the fitness of the alleles from pollen in diploid genotypes be 1 and $1-r_{\mathrm{P}} S_{\mathrm{P}}$ for $A$ and $a$, and similarly 1 and $1-r_{\mathrm{O}} s_{\mathrm{O}}$ for the alleles $A$ and $a$ from ovules. $r_{\mathrm{O}}$ and $r_{\mathrm{P}}$ are the ratios of selection coefficients between the gametophyte and sporophyte stages. These two parameters have the same meaning as the parameter $c$ of Charlesworth and Charlesworth (1992a) that denotes a factor for obtaining the selection coefficient for the sporophyte stage, given the selection coefficient in the pollen stage. Therefore, the four ordered genotype fitnesses are $w_{11}=1$ for $A A, w_{12}=1-r_{\mathrm{O}} s_{\mathrm{O}}$ for $A a$, $w_{21}=1-r_{\mathrm{P}} S_{\mathrm{P}} \quad$ for $a A$, and $w_{22}=1-r_{\mathrm{O}} s_{\mathrm{O}}-r_{\mathrm{P}} s_{\mathrm{P}}+$ $O\left(s_{\mathrm{O}} s_{\mathrm{P}}\right)$ for $a a$. Note that a weak selection is assumed so that all items containing the second or higher order of selection coefficient are ignored. After selection at the sporophyte stage, the genotype frequency in the adult population, $P_{i j}(t+1)$, becomes

$$
P_{i j}(t+1)=w_{i j} P_{i j . S}^{* *}(t+1) / \bar{w}
$$

where $\bar{w}=\sum_{i} \sum_{j} w_{i j} P_{i j . S}^{* *}(t+1)$. Putting equations (1)-(4) into $p(t+1)=P_{11}(t+1)+\left(P_{12}(t+1)+P_{21}(t+1)\right) / 2$, the allele frequency in adult generation $t+1$ can be written as

$$
\begin{aligned}
p(t+1)= & p(t)-\left(m_{\mathrm{S}}+\frac{1}{2}(1-\alpha) m_{\mathrm{P}}\right)(p(t)-Q) \\
& +\left((1-\alpha)\left(s_{\mathrm{O}}+s_{\mathrm{P}}\right)\right. \\
& \left.+(1+\alpha)\left(r_{\mathrm{O}} s_{\mathrm{O}}+r_{\mathrm{P}} s_{\mathrm{P}}\right)\right) p(t) q(t) / 2 \\
& +\frac{1}{8} \alpha\left(\left(s_{\mathrm{O}}+s_{\mathrm{P}}\right)-\left(r_{\mathrm{O}} s_{\mathrm{O}}+r_{\mathrm{P}} s_{\mathrm{P}}\right)\right) H(t) \\
& +O\left(s_{\bullet}^{2}, m_{\bullet}^{2}, s_{\bullet} m_{\bullet}\right)
\end{aligned}
$$

where $H(t)=P_{12}(t)+P_{21}(t)$, the proportion of heterozygote genotypes in the population. $H(t)$ is a recurrence equation of the form

$$
\begin{aligned}
H(t+1)= & \frac{1}{2} \Delta \alpha H(t)+2 m_{\mathrm{S}}(1-F) Q(1-Q) \\
& +(1-\alpha)\left(2 p(t) q(t)\left(\Delta-\left(r_{\mathrm{O}} s_{\mathrm{O}}+r_{\mathrm{P}} s_{\mathrm{P}}\right) / 2\right)\right. \\
& +(p(t)-q(t))\left(m_{\mathrm{P}}(p(t)-Q)\right. \\
& \left.\left.-p(t) q(t)\left(s_{\mathrm{O}}+s_{\mathrm{P}}\right)\right)\right) \\
& +O\left(s_{\bullet}^{2}, m_{\bullet}^{2}, s_{\bullet} m_{\bullet}\right)
\end{aligned}
$$

where $\Delta=1-m_{\mathrm{S}}+\left(r_{\mathrm{O}} s_{\mathrm{O}}+r_{\mathrm{P}} s_{\mathrm{P}}\right)(q(t)-\alpha / 4)$. Thus, the migration load, measured at the sporophyte stage at generation $t+1$ in adult and denoted by $L(=1-\bar{w})$, is

$$
L \approx\left(r_{\mathrm{O}} s_{\mathrm{O}}+r_{\mathrm{P}} s_{\mathrm{P}}\right) q(t)
$$

At steady state $(H(t+1)=H(t)=H$ and $p(t+1)=p(t)=p$, $q=1-p)$, according to equation (6), we can obtain

$$
H=\frac{2(1-\alpha)}{1-\alpha / 2} p q+\Lambda\left(m_{\bullet}, s_{\bullet}\right)+O\left(m_{\bullet}^{2}, s_{\bullet}^{2}, s_{\bullet} m_{\bullet}\right)
$$

where $\Lambda\left(m_{\bullet}, s_{\bullet}\right)$ represents all items containing only the first order of migration rate or selection coefficient. Note that $(1-\alpha) /(1-\alpha / 2)$ in equation (8) is actually equal to $1-F_{i s}$ (Wright, 1969, p 195). Also, the inbreeding coefficient in migrant seeds, $F$, is contained in one of the items in $\Lambda\left(m_{\bullet}, s_{\bullet}\right)$. Without loss of generality, let $s_{\mathrm{O}} / s_{\mathrm{P}}=K_{s}$ and $r_{\mathrm{O}} / r_{\mathrm{P}}=K_{r}$, where $s_{\mathrm{P}}$ is assumed not to be zero. The biological meanings of these parameters are very clear. For instance, when $K_{s}>1$, selection is stronger in ovules than in pollen grains; when $K_{r}>1$, the change in the selection coefficient between the gametophyte and sporophyte stages is greater for the alleles from ovules than from pollen.

Substituting equation (8) into equation (5), we can derive the analytical expression for allele frequency at steady state, $q$. Thus, the general analytical expression for migration load can be obtained by replacing $q(t)$ in equation (7) with $q$ at steady state,

$$
\begin{aligned}
L= & \frac{1}{2}\left(1+\tilde{m} / s_{\mathrm{T}}\right)\left(K_{s} K_{r}+1\right) r_{\mathrm{P}} s_{\mathrm{P}} \\
& \times\left(1-\left(1-\frac{4 \tilde{m}(1-Q)}{s_{\mathrm{T}}\left(1+\tilde{m} / s_{\mathrm{T}}\right)^{2}}\right)^{1 / 2}\right)
\end{aligned}
$$

where $s_{\mathrm{T}}=(1 /(2-\alpha))\left((1-\alpha)\left(K_{s}+1\right)+\left(K_{s} K_{r}+1\right) r_{\mathrm{P}}\right) s_{\mathrm{P}}$, and $\tilde{m}=m_{\mathrm{S}}+((1-\alpha) / 2) m_{\mathrm{P}}$.

Note that the inbreeding coefficient $F$ from migrant seeds is not included in equation (9) because all the items containing $F$ in the analytical expression of $q$ are ignorable $\left(O\left(m_{S} s_{\bullet}\right)\right)$.

According to equation (9), we can see that migration load is slightly increased with pollen flow when the selection coefficient is greater at the gametophyte stage than that at the sporophyte stage, but substantially increased otherwise (Figure 1). The migration load is increased with seed flow in the cases of either small or large strengths of selection against alleles from ovules (Figure 2). Seed flow is more effective than pollen flow in increasing the migration load, which can also be directly proved from equation (9), that is $\partial L / \partial m_{\mathrm{S}}=2\left(\partial L / \partial m_{\mathrm{P}}\right)$. In addition, the migration load of pollen grains can be significantly changed with the type of mating system. The migration load can be seriously affected by pollen 


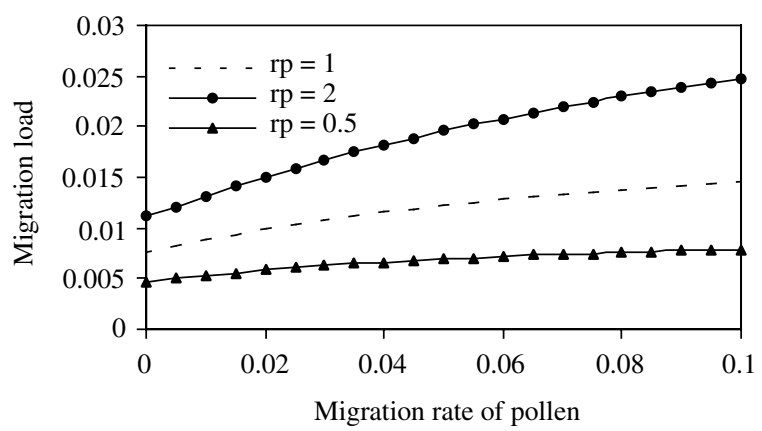

Figure 1 Effects of pollen flow on the migration load in the local population under a balance between migration and selection. Results are obtained according to equation (9) in the text. The ratio of selection coefficients between the gametophyte and the sporophyte stages for allele $a$ from pollen, $r_{\mathrm{P}}$, is independently set at 1, 2, and $1 / 2$. Other parameters are the ratio of selection coefficient of allele $a$ from ovule to that from pollen $\left(K_{\mathrm{s}}\right)=1.0$; the ratio of $r_{\mathrm{O}}$ to $r_{\mathrm{P}}$ $\left(K_{\mathrm{r}}\right)=1.0$, where $r_{\mathrm{O}}$ is the ratio of selection coefficients between the gametophyte and the sporophyte stages for allele $a$ from ovule; the selection coefficient of allele $a$ in pollen $\left(s_{P}\right)=0.02$; selfing rate $(\alpha)=0.05$; the frequency of allele $A$ in migrants $(Q)=0.5$; and migration rate of seed $\left(m_{\mathrm{S}}\right)=0.02$.
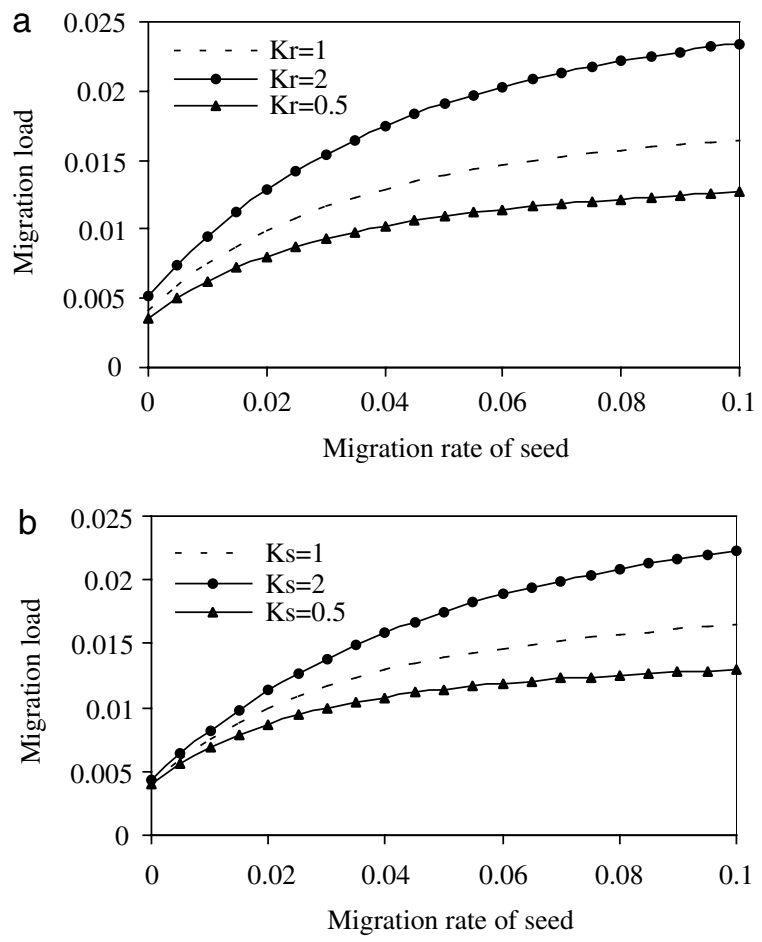

Figure 2 Effects of seed flow on the migration load in the local population under a balance between migration and selection. Results are obtained according to equation (9) in the text. In (a) the ratio of $r_{\mathrm{O}}$ to $r_{\mathrm{P}}\left(K_{\mathrm{r}}\right)$ is independently set at 1,2 , and $1 / 2$, where $r_{\mathrm{O}}$ and $r_{\mathrm{P}}$ are the ratios of selection coefficients between the gametophyte and the sporophyte stages for allele $a$ from ovule and pollen, respectively. The ratio of selection coefficient of allele $a$ from ovule to that from pollen $K_{\mathrm{s}}$ is fixed at 1.0. In (b) $K_{\mathrm{s}}$ is independently set at 1,2 , and $1 / 2$, and $K_{\mathrm{r}}$ is fixed at 1.0. Other common parameters are the selection coefficient of allele $a$ from pollen $\left(s_{P}\right)=0.02$, migration rate of pollen $\left(m_{\mathrm{P}}\right)=0.02$, selfing rate $(\alpha)=0.05$, the frequency of allele $A$ in migrants $(Q)=0.5$, and $r_{\mathrm{P}}=1.0$.

flow in predominantly outcrossing populations, while there is little effect in highly selfing populations (Figure 3).

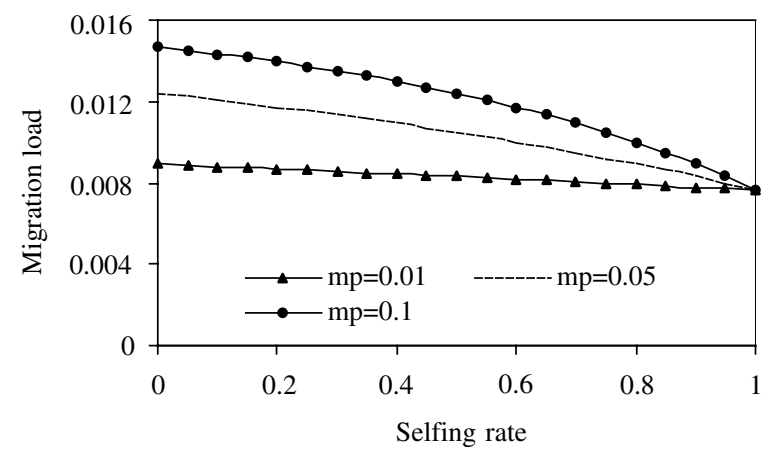

Figure 3 Effects of mating systems on the migration load in the local population under a balance between migration and selection. Results are obtained according to equation (9) in the text. Migration rate of pollen $\left(m_{\mathrm{P}}\right)$ is independently set at $0.01,0.05$, and 0.1 . Other parameters are the ratio of selection coefficients between the gametophyte and the sporophyte stages for allele $a$ from pollen $\left(r_{\mathrm{P}}\right)=1.0$; the ratio of $r_{\mathrm{O}}$ to $r_{\mathrm{P}}\left(K_{\mathrm{r}}\right)=1.0$, where $r_{\mathrm{O}}$ is the ratio of selection coefficients between the gametophyte and the sporophyte stages for allele $a$ from ovule; the ratio of selection coefficient of allele $a$ from ovule to that from pollen $\left(K_{\mathrm{s}}\right)=1.0$; the frequency of allele $A$ in migrants $(Q)=0.5$; and the selection coefficient of allele $a$ in pollen $\left(s_{\mathrm{P}}\right)=0.02$.

Denote by $\lambda$ the ratio of total selection coefficient at the gametophyte versus the sporophyte stages, that is, $\lambda=\left(K_{s}+1\right) s_{\mathrm{P}} /\left(K_{s} K_{r}+1\right) r_{\mathrm{P}} s_{\mathrm{P}}$. According to equation (9), the general expression for migration load can be approximated under various ratios of migration rate $(\tilde{m})$ to selection coefficient $\left(s_{\mathrm{T}}\right)$ (Table 1). Migration load can be a linear function of migration rate only when the migration rate is much smaller than $\tilde{m} \ll s_{\mathrm{T}}$, or approximately equal to $\left(\tilde{m} \approx s_{\mathrm{T}}\right)$, the selection coefficient. In contrast, migration load is almost unrelated to migration rate when the migration rate is much greater than the selection coefficient $\left(\tilde{m} \gg s_{T}\right.$; Table 1$)$.

In the case of complete outcrossing $(\alpha=0)$ or selfing $(\alpha=1)$, the analytic expression for migration load can be readily simplified (Table 1 ). In particular, when $\lambda=0$ (no selection at the gametophyte stage) and $\alpha=0$, the results reduce to those obtained by Wright (1977, p 486).

\section{Random processes}

To consider the effect of genetic drift, the distribution of gene frequency must be calculated. For simplicity, we consider the case of complete outcrossing, that is, $\alpha=0$. According to equation (5) the average change in gene frequency, $M_{\delta p}$, can be obtained as $M_{\delta p}=$ $-\tilde{m}(p-Q)+s_{\mathrm{T}} p q$, where $\tilde{m}=m_{\mathrm{S}}+m_{\mathrm{P}} / 2$. The variance for the change of allele frequency, $V_{\delta p}$, is $V_{\delta p} \approx$ $p(1-p) / 2 N$, where $N$ is the effective population size. Direct use of Wright's formula (1969, p 361) yields the density distribution, $\phi(p)=\mathrm{Ce}^{4 N s_{\mathrm{T}} p} p^{4 N \tilde{m} Q-1}$ $(1-p)^{4 N \tilde{m}(1-Q)-1}$, where $C$ is a constant that satisfies $\int_{0}^{1} C \phi(p) \mathrm{d} p=1$.' Therefore, the average migration load is

$$
\bar{L}=\int_{0}^{1} \frac{2 s_{\mathrm{T}}}{\lambda+1}(1-p) \phi(p) \mathrm{d} p / \int_{0}^{1} \phi(p) \mathrm{d} p
$$

The average migration load at steady state changes with the population size, and small populations have large migration loads (Figure 4). 
Table 1 The approximate analytical formulae for the migration load under various ratios of selection coefficient $\left(s_{\mathrm{T}}\right)$ to migration rate $(\tilde{m})$. $\alpha$ is the selfing rate, $Q$ is the frequency of allele $A$ in migrants, and $\lambda$ is the ratio of total selection coefficient between the gametophyte and the sporophyte stages

\begin{tabular}{lllr}
\hline Mating systems & $\tilde{m} \ll \mathrm{s}_{T}$ & $\tilde{m} \approx \mathrm{s}_{T}$ & $\tilde{m} \gg \mathrm{s}_{T}$ \\
\hline Partial selfing & $\frac{2-\alpha}{(1-\alpha) \lambda+1} \tilde{m}(1-Q)$ & $\frac{2-\alpha}{(1-\alpha) \lambda+1} \tilde{m}(1-\sqrt{Q})$ & $\frac{2-\alpha}{(1-\alpha) \lambda+1} s_{\mathrm{T}}(1-Q)$ \\
Selfing & $m_{\mathrm{S}}(1-Q)$ & $m_{\mathrm{S}}(1-\sqrt{Q})$ & $s_{\mathrm{T}}(1-Q)$ \\
Complete outcrossing & $\frac{2}{\lambda+1} \tilde{m}(1-Q)$ & $\frac{2}{\lambda+1} \tilde{m}(1-\sqrt{Q})$ & $\frac{2}{\lambda+1} s_{\mathrm{T}}(1-Q)$ \\
\hline
\end{tabular}
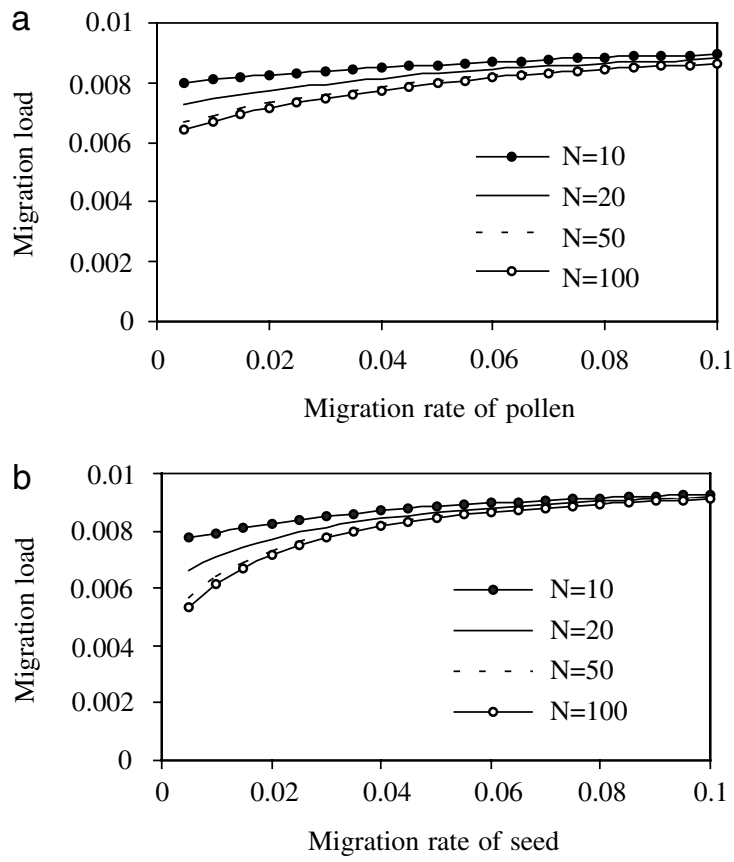

Figure 4 Effects of genetic drift on the migration load. Results are obtained according to equation (10) in the text. Effective population size $(N)$ is independently set at $10,20,50$, and 100 . In (a) migration rate of seed $\left(m_{\mathrm{S}}\right)$ is fixed at 0.02 . In (b) migration rate of pollen $\left(m_{\mathrm{P}}\right)$ is fixed at 0.02 . Other common parameters are: the compound selection coefficient $\left(s_{\mathrm{T}}\right)=0.02$, the ratio of total selection coefficient between the gametophyte and the sporophyte stages $(\lambda)=1.0$, and the frequency of allele $A$ in migrants $(Q)=0.5$.

\section{Discussion}

The theoretical originality of the present model of migration load is that it considers the joint effects of pollen and seed flow together with both gametophyte and sporophyte selection. The relations between migration load and seed and pollen flow are explicitly formulated. Since Wright's model of migration load (Wright, 1969) is very general and valid only for the case where diploid migrants and the selection at the sporophyte stage occur, the present model can be seen as a significant extension of theory. Also, because of the incorporation of seed flow and ovule selection, the present model significantly differs from the previous model of mutation load (Charlesworth and Charlesworth, 1992a) and the phenotypic model of pollen competition (Lankinen and Skogsmyr, 2001).
The results presented here show that under certain conditions, migration load of large magnitude can be maintained in a local population by seed and pollen flow. Our results indicate that when the selection coefficient is greater at the gametophyte stage than that at the sporophyte stage, the migration load measured at the sporophyte stage is not substantially increased by pollen flow. The selection coefficient at the sporophyte stage is critical in determining the role of pollen flow in the reduction of local population fitness. Thus, the indirect effects through selection at the sporophytic stage are important in understanding the migration load of pollen grains in addition to the direct effects of pollen selection. In their model of mutation load, Charlesworth and Charlesworth (1992a) also showed that strong pollen selection is usually expected to increase sporophyte fitness only slightly.

Although there are many studies on the effects of pollen competition on progeny vigour (eg Mulcahy et al, 1996; Kalla and Ashman, 2002), the results remain controversial because such effects are not always evident (see Mitchell, 1997). Furthermore, normal pollen competition in natural populations does not mean the complete purging of detrimental alleles. However, if the strength of pollen selection is very strong, much greater than that at the sporophyte stage, detrimental alleles in migrant pollen grains can be effectively prevented from forming zygotes. In this case, the reduction in fitness is mainly attributable to the detrimental alleles coming from migrant seeds and also from resident ovules if the strength of selection is much smaller in ovules than in pollen grains at the gametophyte stage in the local population. Thus, the migration load of seeds remains present as long as seed flow takes place.

The relative migration loads of pollen versus seeds are closely associated with the distinct biological paths through which seed and pollen flow are effected. Our results show that migration load depends more on seed than pollen flow. When both migration rates are the same, the migration load of seed is greater than that of pollen. In practice, the relative migration loads of seeds versus pollen grains vary with species or populations of the same species. Burt (1995) calculated the relative change in fitness as the change in the natural logarithm (ie $\Delta \bar{w}=\bar{w} \cdot \Delta \ln \bar{w}$ ), and found that pollen flow can decrease fitness by $\sim 0.12$ per generation in Anthoxanthum odoratum. Seed flow can reduce fitness by $\sim 0.0045$ per generation in Impatiens capensis. In Ipomopsis aggregata, seed and pollen flow can reduce fitness by $\sim 0.002$ and $\sim 0.0008$ per generation, respectively.

Our results also give the theoretical conditions under which a great care must be taken when the ratio of 
$m_{\mathrm{P}} / 2 m_{\mathrm{S}}$ is used to assess quantitatively the relative migration loads of pollen versus seeds in predominantly outcrossing populations. Such an estimate is valid only when the migration rate $(\tilde{m})$ is much smaller than the selection coefficient $\left(s_{\mathrm{T}}\right)$. Theoretically, estimate of $m_{\mathrm{P}} / 2 m_{\mathrm{S}}$ can be calculated using genetic markers with different modes of inheritance (biparental versus maternal versus paternal inheritances; Ennos, 1994; $\mathrm{Hu}$ and Ennos, 1997, 1999), and this method has been demonstrated with several outcrossing and inbreeding species (Ennos, 1994). Many tree species with wind pollination and animal dispersal of seeds, such as Quercus petraea/ $Q$. robur, have a large pollen/seed migration ratio (Ennos, 1994), implying that any migration load, is mainly caused by pollen. Other plant species, such as Argania spinosa, with insect pollination and ruminant dispersal of seeds, have a small pollen/seed migration ratio (Ennos et al, 1999), implying that seed and pollen flow have comparable migration loads. Thus, it can be predicted that migration load is mainly caused by pollen grains rather than by seeds in many predominantly outcrossing species. In contrast, migration load is mainly brought about by seeds rather than by pollen grains in many predominately selfing species.

The relative magnitude in the strength of selection between the gametophyte and the sporophyte stages $(\lambda)$ is important since it is also associated with the indirect effect of pollen (including migrants) or ovule selection on the population fitness at the sporophyte stage. In their model of mutation load Charlesworth and Charlesworth (1992a) limited the strength of selection in pollen to that in the sporophyte, while Mulcahy et al (1996) thought that the strength of selection could also be greater at the gametophyte stage than that at the sporophyte stage. Theoretically, the consequences of either case can be examined by altering the relevant parameters (eg Figure 1). Moreover, our model suggests a way to roughly estimate this ratio, that is,

$$
\hat{\lambda}=\frac{\hat{\tilde{m}}(1-\hat{Q})(2-\hat{\alpha})-1}{(1-\hat{\alpha}) \hat{L}}
$$

when $\tilde{m} \ll s_{\mathrm{T}}$ or

$$
\hat{\lambda}=\frac{\hat{\tilde{m}}(1-\sqrt{\hat{Q}})(2-\hat{\alpha})-1}{(1-\hat{\alpha}) \hat{L}}
$$

when $\tilde{m} \approx s_{\mathrm{T}}$.

The precondition of the above method is that migration load $(\hat{L})$ and other parameters $(\hat{\alpha}, \hat{\tilde{m}}$, and $\hat{Q})$ should be estimated beforehand using other methods. For example, a reduction in mean fitness $(\hat{L})$ because of migration of seed or pollen can be estimated with transplantation experiments (Burt, 1995).

Although the present model extends Wright's theory of migration load in several aspects, it is important to note our assumption on the strength of natural selection in this study. A major limitation is that only weak selection is considered either at the gametophyte stage or at the sporophytic stage. Since small migration rates of pollen and seeds are considered, this accordingly requires weak selection theory; otherwise, a balance between selection and migration cannot be reached. However, this assumption could be violated in practice.
Pollen or ovule selection is in some cases very strong so that inbreeding or outbreeding depression can be substantially purged (eg Kärkkäinen and Savolainen, 1993). Furthermore, interactions of pollen by pollen or pollen by style are also recorded (Landi and Frascaroli, 1988; Cruzan, 1990; see review by Mulcahy et al, 1996), which likely generates a more complicated selection system. Therefore, a challenge for the future is to develop techniques that incorporate epistatic selection into the model, so that more sophisticated insights into the migration load attributable to seeds and pollen grains can be obtained.

\section{Acknowledgements}

We greatly appreciate Dr John Brookfield, the designated editor, and two anonymous referees for very helpful comments and corrections that substantially improved the early version of this article. Many thanks are also given to Dr D O'Malley for discussion

\section{References}

Barton NH (2001). The evolutionary consequences of gene flow and local adaptation: future approaches. In: Clobert J, Danchin E, Dhondt AA, Nichols JD (eds) Dispersal, Oxford University Press: New York. pp 329-340.

Barton NH, Gale KS (1993). Genetic analysis of hybrid zones. In: Harrison RG (ed) Hybrid Zones and the Evolutionary Process, Oxford University Press: New York. pp 13-45.

Barton NH, Whitlock MC (1997). The evolution of metapopulations. In: Hanski IA, Gilpin ME (eds) Metapopulation Biology: Ecology, Genetics, and Evolution, Academic Press: San Diego. pp 183-210.

Björkman T (1995). The effect of pollen load and pollen grain competion on fertilization success and progeny performance in Fagopyrum esculentum. Euphytica 83: 47-52.

Burt A (1995). The evolution of fitness. Evolution 49: 1-8.

Casper BB (1988). Evidence for selective embryo abortion in Cryptantha flava. Am Nat 132: 318-326.

Charlesworth D (1989a). Why do plants produce so many more ovules than seeds? Nature 338: 21-22.

Charlesworth D (1989b). Evolution of low female fertility in plants: pollen limitation, resource allocation and genetic load. Trends Ecol Evol 4: 289-292.

Charlesworth D, Charlesworth B (1987). Inbreeding depression and its evolutionary consequences. Ann Rev Ecol Syst 18 237-268.

Charlesworth D, Charlesworth B (1992a). The effects on genetic load of selection in the gametophyte stage. In: Ottaviano E, Mulcahy DL, Gorla MS, Mulcahy GB (eds) Angiosperm Pollen and Ovules, Springer-Verlag: Berlin. pp 401-407.

Charlesworth D, Charlesworth B (1992b). The effects of selection in the gametophyte stage on mutational load. Evolution 46: 703-720.

Cruzan MB (1990). Pollen-pollen and pollen-style interactions during pollen tube growth in Erythronium grandiforum (Liliaceae). Am J Bot 77: 116-122.

Ellstrand NC, Prentice HC, Hancock JF (1999). Gene flow and introduction from domesticated plants into their wild relatives. Ann Rev Ecol Systems 30: 539-563.

Ennos RA (1994). Estimating the relative rates of pollen and seed migration among plant populations. Heredity $\mathbf{7 2}$ 250-259.

Ennos RA, Sinclair WT, Hu XS, Langdon A (1999). Using organelle markers to elucidate the history, ecology and evolution of plant populations. In: Hollingsworth PM, Bateman RM, Gornall RJ (eds) Molecular Systematics and Plant Evolution. Taylor \& Francis: London. pp 1-19. 
Haldane JBS (1932). The time of action of genes, and its bearing on some evolutionary problems. Am Nat 66: 5-24.

$\mathrm{Hu}$ XS, Ennos RA (1997). On estimation of the ratio of pollen to seed flow among plant populations. Heredity 79: 541-552.

$\mathrm{Hu}$ XS, Ennos RA (1999). Impacts of seed and pollen on population differentiation for plant genomes with three contrasting modes of inheritance. Genetics 152: 441-450.

Kalla SE, Ashman TL (2002). The effects of pollen competition on progeny vigor in Fragaria virginiana (Rosaceae) depend on progeny growth environment. Int J Plant Sci 163: 335-340.

Kärkkäinen K, Savolainen O (1993). The degree of early inbreeding depression determines the selfing rate at the seed stage: model and results from Pinus sylvestris (Scots pine). Heredity 71: 160-166.

Landi PA, Frascaroli E (1988). Pollen-style interactions in Zea mays L. In: Cresti M, Gori P, Pacini E (eds) Sexual Reproduction in Higher Plants, Springer: Berlin, Heideberg, New York. pp 315-320.

Lankinen A, Skogsmyr I (2001). The effect of pollen competition on maintenance of variation in fertilisation ability. Oikos 93: 459-469.

Lenormand $\mathrm{T}$ (2002). Gene flow and the limits to natural selection. Trends Ecol Evol 17: 183-189.

Melser C, Klinkhamer PGL (2001). Selective seed abortion increases offspring survival in Cynoglossum officinale (Boraginaceae). Am J Bot 88: 1033-1040.

Mitchell RJ (1997). Effects of pollen quantity on progeny vigor: evidence from the desert mustard Lesquerella fendleri. Evolution 51: 1679-1684.

Mulcahy DL, SariGorla M, Mulcahy GB (1996). Pollen selectionpast, present and future. Sex Plant Reprod 9: 353-356.
Niesenbaum RA (1999). The effects of pollen load size and donor diversity on pollen performance, selective abortion, and progeny vigor in Mirabilis jalapa (Nyctaginaceae). Am J Bot 86: 261-268.

Quesada M, Winsor JA, Stephenson AG (1996). Effects of pollen competition on the reproductive performance in cucurbit hybrids (Cucurbitaceae): F1 and backcross generations. Can J Bot 74: 1113-1118.

Rocha OJ, Stephenson AG (1991). Effects of nonrandom seed abortion on progeny performance in Phaseolus coccineus L. Evolution 45: 1198-1208.

Snow AA, Spira TP (1991). Pollen vigor and the potential for sexual selection in plants. Nature 352: 796-797.

Snow AA, Spira TP (1996). Pollen-tube competition and male fitness in Hibiscus moscheutos. Evolution 50: 1866-1870.

Sorensen FC (1982). The role of polyembryonal vitality in the genetic system of conifers. Evolution 36: 725-733.

Stephenson AG, Winsor JA. (1986). Lotus corniculatus regulates offspring quality through selective fruit abortion. Evolution 40: 453-458.

Waser NM, Price MV (1989). Optimal outcrossing in Ipomopsis aggregata: seed set and offspring fitness. Evolution 43: 1097-1109.

Whitlock MC (2002). Selection, load and inbreeding depression in a large metapopulation. Genetics 160: 1191-1202.

Wright S (1969). Evolution and the Genetics of Populations, Vol. 2, The Theory of Gene Frequencies, University Chicago Press: Chicago.

Wright S (1977). Evolution and the Genetics of Populations. Vol. 3, Experimental Results and Evolutionary Deductions, The University of Chicago Press: Chicago. 